\title{
Lymphadenectomy in women with endometrial cancer: aspiration and reality from a radiation oncologist's point of view

Robert Foerster ${ }^{*}$, Robert Kluck, Nathalie Arians, Stefan Rieken, Harald Rief, Sebastian Adeberg, Tilman Bostel, Ingmar Schlampp, Juergen Debus and Katja Lindel

\begin{abstract}
Background: To investigate the meaning of lymphadenectomy (LNE) in women with endometrial cancer (EC) for clinical outcome and secondly to determine the impact of the method of adjuvant radiotherapy (RT) on survival as well as to define prognostic factors.
\end{abstract}

Methods: 322 patients (pts) underwent adjuvant RT for endometrioid EC at our department from 2004 until 2012 and were included in this retrospective study. Chi-square test, LogRank test and Cox regression were used for statistical analyses.

Results: Median age at diagnosis: 66 years. FIGO stages: FIGO I 69.4 \%, FIGO II 15.3 \%, FIGO III 14.5 \%, FIGO IV 0.9 \%. Surgical staging: $30.6 \%$ pelvic/paraaortic LNE, $45 \%$ sole pelvic LNE, $8.8 \%$ sampling of suspicious lymph nodes, $15.6 \%$ no LNE. Adjuvant chemotherapy (ChT): $3.2 \%$. Sole intravaginal brachytherapy (IVB): $60.2 \%$. IVB + external beam radiotherapy (EBRT): $39.8 \%$. 5-year local recurrence free survival (LRFS): $90.6 \%$, distant metastases free survival (DMFS): $89.8 \%$, overall survival (OS):79.3\%. In multivariate analysis age $(p=.007)$, pT stage $(p=.029)$, lymph node status ( $p=.003$ ), grading ( $p=.011$ ) and lymphovascular space invasion (LVSI; $p=.008$ ) remained as independent prognostic factors for OS. Resection status $(p=.01)$ and LVSI $(p=.014)$ were independent prognostic factors for LRFS and LVSI $(p=.008)$ was the only independent prognostic factor for DMFS. There was no statistically significant survival benefit from LNE in LRFS $(p=.561)$, DMFS $(p=.981)$ or OS $(p=.791)$. 5 -year LRFS in stage I and II: 96.0 and $82.9 \%$ after sole IVB, 90.8 and $81.6 \%$ after combined IVB/EBRT $(p=.105 ; p=.970) .5$-year OS rates for stage I and II: 86.5 and $71.3 \%$ after sole IVB, $84.2 \%$ and $69.2 \%$ after combined IVB/EBRT ( $p=.153 ; p=.619$ ).

Conclusion: Comprehensive surgical staging is rarely performed and may be omitted in women with endometrioid EC in stages I-II. Sole IVB delivers equally good local control as combined IVB/EBRT in pts with FIGO stage I and II disease. LVSI deserves more attention as a prognostic factor and these pts may require a combined local and systemic therapy.

Keywords: Adjuvant radiotherapy, Endometrial cancer, Lymphadenectomy, Prognostic factors, Survival

\footnotetext{
* Correspondence: robert.foerster@med.uni-heidelberg.de

Department of Radiation Oncology, University Hospital Heidelberg, Im

Neuenheimer Feld 400, 69120 Heidelberg, Germany
} 


\section{Background}

Endometrial cancer (EC) is the most common gynecologic malignancy. While therapy guidelines are widely established and the prognosis is generally favorable, optimal treatment remains controversial. In particular the conductance of systematic lymphadenectomy (LNE) and the role of external beam radiotherapy (EBRT) in early stages have been actively disputed, since both therapy modalities are known to cause substantial morbidity. Current guidelines recommend systematic pelvic and paraaortic LNE with investigation of at least 15 pelvic and 10 paraaortic lymph nodes from FIGO IB onward $[1,2]$. However, clinical practice differs among surgical centers and many patients are spared LNE or only undergo sampling of suspicious pelvic lymph nodes commonly omitting paraaortic lymph nodes. For radiation oncologists it can be challenging to recommend the appropriate adjuvant therapy for these patients (pts), especially when they present with additional risk factors, such as grade-3- histology or lymphovascular space invasion (LVSI). We therefore designed this retrospective analysis to elucidate the clinical outcome in the pts treated with adjuvant radiotherapy (RT) at our department and further to investigate the role of LNE and known prognostic factors.

\section{Methods}

Between 2004 and 2012 we performed adjuvant RT in 322 women with endometrioid EC at our department. All pts were included in this retrospective study, which was approved by the ethics committee of the University of Heidelberg. Due to its retrospective and blinded design consent was not required. By revision of the electronic patient charts we collected detailed information on stage, grading, resection status, LVSI, primary surgical therapy, adjuvant RT and additional adjuvant chemotherapy (ChT). FIGO 2009 classification was used for staging and patients were reclassified if necessary. Survival analysis was done for local recurrence free survival (LRFS), distant metastases free survival (DMFS) and overall survival (OS). LRFS was considered to be the time between first diagnosis and first recurrence within the irradiation field. DMFS was calculated as the time from first diagnosis until distant relapse. OS was calculated from date of first diagnosis until death from any cause. Survival was plotted according to Kaplan and Meier. The Log-rank test was used for univariate analysis and Cox proportional hazard model was used for multivariate analysis. The Chi-square test was used to illustrate heterogeneity among treatment groups. A p-value $\leq .05$ was considered statistically significant. Statistical analysis was performed with SPSS 22.0 for Windows.

\section{Results}

Patients' and tumor characteristics

Pts were first diagnosed with EC at a median age of 66 years (range: $36-92)$. FIGO stages were distributed as follows: stage I $69.4 \%$, stage II $15.3 \%$, stage III $14.5 \%$, stage IV $0.9 \%$. Positive lymph nodes (N1) were found in $9.7 \%, 24.2 \%$ had an undifferentiated tumor grading (G3) and $16.5 \%$ showed LVSI. Resection was incomplete (R2) in $0.3 \%$, microscopically positive resection margins (R1) were found in $2.2 \%$ and resection status was indeterminable $(\mathrm{Rx})$ in $5.0 \%$ (Table 1 ).

Primary surgical therapy consisted of hysterectomy and bilateral salpingo-oophorectomy in all patients. Pelvic and paraaortic LNE was conducted in 98 pts (30.6\%), 144 (45.0\%) received sole pelvic LNE, 28 (8.8\%) only underwent sampling of suspicious lymph nodes and in 50 (15.6\%) LNE was omitted. RT consisted of EBRT in 1.8 - 2.0 Gy fractions to a cumulative dose of 40.0 - 54.0 Gy and / or high dose rate (HDR) intravaginal brachytherapy (IVB) in 5.0 - 5.5 Gy fractions to a cumulative dose of 10.0-22.0 Gy. One hundred twenty eight (39.8\%) pts received combined IVB/EBRT and 194 $(60.2 \%)$ received IVB alone. In the IVB/EBRT group the median total EBRT dose was $45.0 \mathrm{~Gy}$ and the median total IVB dose was 10.0 Gy. Patients in the sole IVB group received a median total dose of 22.0 Gy. Additional adjuvant ChT consisting of 6 cycles of carboplatin and paclitaxel was given to $3.1 \%(n=10)$ before or after adjuvant RT. Only patients with stage III and IV disease received ChT. A detailed overview on surgical lymph node staging and radiotherapy for FIGO stages I-IV as well as for FIGO stage I according to risk stratification [3] is given in Table 2. Patients with intermediate risk were statistically significantly more often treated with combined EBRT/IVB when surgical lymph node staging was omitted ( $p=.009$, Table 2$)$.

\section{Survival analysis}

Median follow-up was 49.5 months. Sixty-six (20.5 \%) pts died during follow-up, 27 (8.4\%) had a local recurrence and $26(8.1 \%)$ developed distant metastases. One $(0.3 \%)$ patient presented with synchronous distant metastases to the cervical lymph nodes at first diagnosis of endometrioid EC. LRFS, DMFS and OS after 5 years were 90.6, 89.8 and $79.3 \%$ respectively (Fig. 1). Sites of recurrences are shown in Table 3 .

\section{Univariate analysis}

Local control was worse with higher pT stage $(p=.001)$, positive regional lymph nodes $(p<.001)$, positive or indeterminable resection margins $(p<.001)$ and LVSI $(p=.001$; Fig. 2)

Distant metastases occurred earlier in pts with higher FIGO stage $(p=.001)$, higher pT stage $(p<.001)$ and 
Table 1 Patients' and tumor characteristics

\begin{tabular}{|c|c|c|}
\hline \multicolumn{3}{|c|}{ Age $N=322$} \\
\hline \multirow{3}{*}{$\begin{array}{l}\text { median } \\
\text { range }\end{array}$} & \multicolumn{2}{|c|}{66 years } \\
\hline & \multicolumn{2}{|c|}{$36-92$ years } \\
\hline & $n$ & $\%$ \\
\hline \multicolumn{3}{|c|}{$\mathrm{FIGON}=320$} \\
\hline $\mid A$ & 104 & $32.5 \%$ \\
\hline IB & 118 & $36.9 \%$ \\
\hline$\|$ & 49 & $15.3 \%$ \\
\hline$\| \mathrm{A}$ & 13 & $4.1 \%$ \\
\hline$\| \mathrm{B}$ & 4 & $1.3 \%$ \\
\hline$\| \mathrm{IIC}$ & 29 & $9.1 \%$ \\
\hline IVA & 2 & $0.6 \%$ \\
\hline IVB & 1 & $0.3 \%$ \\
\hline \multicolumn{3}{|c|}{ pT stage $N=320$} \\
\hline T1 & 237 & $74.1 \%$ \\
\hline T2 & 58 & $18.1 \%$ \\
\hline T3 & 24 & $7.5 \%$ \\
\hline T4 & 1 & $0.3 \%$ \\
\hline \multicolumn{3}{|c|}{ Nodal status $N=320$} \\
\hline No & 289 & $90.3 \%$ \\
\hline N1 & 31 & $9.7 \%$ \\
\hline \multicolumn{3}{|c|}{ Grading $N=322$} \\
\hline G1 & 69 & $21.4 \%$ \\
\hline G2 & 175 & $54.3 \%$ \\
\hline G3 & 78 & $24.2 \%$ \\
\hline \multicolumn{3}{|c|}{ LVSI N= 322} \\
\hline no LVSI & 269 & $83.5 \%$ \\
\hline LVSI & 53 & $16.5 \%$ \\
\hline \multicolumn{3}{|c|}{ Resection N = 322} \\
\hline RO & 298 & $92.5 \%$ \\
\hline R1 & 7 & $2.2 \%$ \\
\hline R2 & 1 & $0.3 \%$ \\
\hline $\mathrm{RX}$ & 16 & $5.0 \%$ \\
\hline \multicolumn{3}{|c|}{ Metastases N = 320} \\
\hline MO & 319 & $99.7 \%$ \\
\hline M1 & 1 & $0.3 \%$ \\
\hline
\end{tabular}

LVSI $(p=.007$; Fig. 2). Age $>66$ years $(p<.045)$, advanced FIGO stage $(p<.001)$, higher $\mathrm{pT}$ stage $(p<.001)$, positive regional lymph nodes $(p<.001)$, higher grading $(p=.001)$, positive or indeterminable resection margins $(p<.001)$ and LVSI $(p<.001$; Fig. 2$)$ were associated with shorter OS. There was no statistically significant survival benefit from LNE in LRFS, DMFS or OS (Fig. 3). Sole IVB was not inferior to combined IVB/EBRT in early stages independent of the conducted surgical lymph
Table 2 Treatment according to (A) FIGO stages and (B) risk stratification

\begin{tabular}{|c|c|c|c|c|}
\hline \multicolumn{2}{|c|}{ (A) FIGO stages } & $n$ & $\%$ & $x^{2}$ \\
\hline \multicolumn{5}{|c|}{$\mathrm{FIGO} \mathrm{I}(N=222)$} \\
\hline \multirow[t]{2}{*}{ LNE } & IVB & 143 & $76.9 \%$ & $p=.194$ \\
\hline & $I V B+E B R T$ & 43 & $23.1 \%$ & \\
\hline \multirow[t]{2}{*}{ No LNE } & IVB & 24 & $66.7 \%$ & \\
\hline & IVB + EBRT & 12 & $33.3 \%$ & \\
\hline \multicolumn{5}{|c|}{$\mathrm{FIGO} \|(N=49)$} \\
\hline \multirow[t]{2}{*}{ LNE } & IVB & 21 & $51.2 \%$ & $p=.478$ \\
\hline & IVB + EBRT & 20 & $48.8 \%$ & \\
\hline \multirow[t]{2}{*}{ No LNE } & IVB & 3 & $37.5 \%$ & \\
\hline & IVB + EBRT & 5 & $62.5 \%$ & \\
\hline \multicolumn{5}{|c|}{$\mathrm{FIGO} \| \mathrm{II}(N=46)$} \\
\hline \multirow[t]{2}{*}{ LNE } & IVB & 3 & $7.2 \%$ & $p=.580$ \\
\hline & IVB + EBRT & 39 & $92.8 \%$ & \\
\hline \multirow[t]{2}{*}{ No LNE } & IVB & 0 & $0 \%$ & \\
\hline & IVB + EBRT & 4 & $100 \%$ & \\
\hline \multicolumn{5}{|c|}{$\mathrm{FIGO} I V(N=3)$} \\
\hline \multirow[t]{2}{*}{ LNE } & IVB & 0 & $0 \%$ & NA \\
\hline & IVB + EBRT & 2 & $100 \%$ & \\
\hline \multirow[t]{2}{*}{ No LNE } & IVB & 0 & $0 \%$ & \\
\hline & IVB + EBRT & 1 & $100 \%$ & \\
\hline \multicolumn{2}{|c|}{ (B) Risk stratification } & $\mathrm{n}$ & $\%$ & $x^{2}$ \\
\hline \multicolumn{5}{|c|}{ Low risk $(N=77)$} \\
\hline \multirow[t]{2}{*}{ LNE } & IVB & 55 & $94.8 \%$ & $p=.988$ \\
\hline & IVB + EBRT & 3 & $5.2 \%$ & \\
\hline \multirow[t]{2}{*}{ No LNE } & IVB & 18 & $94.7 \%$ & \\
\hline & $\mathrm{IVB}+\mathrm{EBRT}$ & 1 & $5.3 \%$ & \\
\hline \multicolumn{5}{|c|}{ Intermediate risk $(N=121)$} \\
\hline \multirow[t]{2}{*}{ LNE } & IVB & 74 & $70.5 \%$ & $p=.009$ \\
\hline & IVB + EBRT & 31 & $29.5 \%$ & \\
\hline \multirow[t]{2}{*}{ No LNE } & IVB & 6 & $37.5 \%$ & \\
\hline & $\mathrm{IVB}+\mathrm{EBRT}$ & 10 & $62.5 \%$ & \\
\hline \multicolumn{5}{|c|}{ High risk $(N=24)$} \\
\hline \multirow[t]{2}{*}{ LNE } & IVB & 14 & $60.9 \%$ & $p=.227$ \\
\hline & IVB + EBRT & 9 & $39.1 \%$ & \\
\hline \multirow[t]{2}{*}{ No LNE } & IVB & 0 & $0 \%$ & \\
\hline & IVB + EBRT & 1 & $100 \%$ & \\
\hline
\end{tabular}

node staging and independent of the risk stratification. Five-year local control in stage I was $96.0 \%$ after sole IVB and $90.8 \%$ after combined IVB/EBRT $(p=.105)$. Five-year OS rates in stage I for sole IVB and combined IVB/EBRT were $86.5 \%$ and $84.2 \%$ respectively $(p=.153)$. For stage II 5 -year LRFS was $82.9 \%$ after sole IVB and $81.6 \%$ after IVB/EBRT $(p=.970)$. Five-year OS 


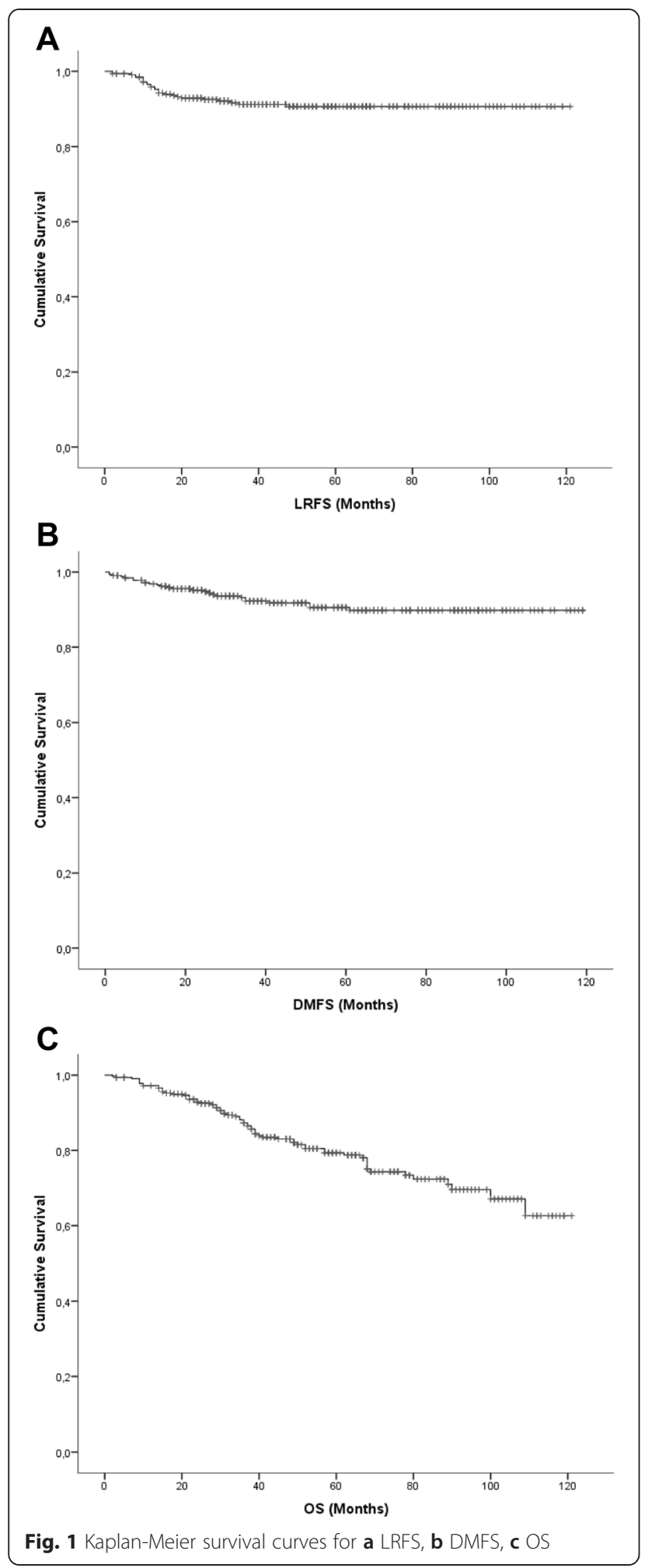

rates in stage II were 71.3 and $69.2 \%$ for the sole IVB and combined IVB/EBRT groups $(p=.619)$. Additional adjuvant ChT did not statistically significantly effect survival in all 3 endpoints.
Table 3 Sites of recurrences

\begin{tabular}{ll}
\hline & $\boldsymbol{n}$ \\
\hline Local recurrences $N=27$ & 8 \\
paraaortic lymph nodes & 12 \\
vaginal pole & 7 \\
pelvic floor and wall & \\
Distant recurrences $N=26$ & 12 \\
lungs & 6 \\
liver & 3 \\
skin & 2 \\
abdominal wall & 3 \\
inguinal lymph nodes & 2 \\
cervical lymph nodes & 2 \\
retroperitoneal lymph nodes & 2 \\
adrenals & 2 \\
intestine & 3 \\
mediastinum & 2 \\
bone & 4 \\
peritoneum & 3 \\
brain & 4 \\
\hline
\end{tabular}

\section{Multivariate analysis}

Age $(p=.017$; HR 1.88 [95 \% CI $1.12-3.16])$, pT stage $(p=.029 ;$ HR 2.13 [1.08-4.21]); lymph node status $(p=.003$; HR 2.74 [95 \% CI 1.40 - 5.37]), grading ( $p=.011$; HR 1.99 [95 \% CI $1.77-3.39])$ and LVSI $(p=.008$; HR 2.22 [95\% CI 1.24-3.98]) were revealed as independent prognostic factors for OS in Cox regression model. LVSI was the only independent prognostic factor $(p=.008$; HR 2.22 [95\% CI 1.24-3.98]) for DMFS. Resection status $(p=.01 ;$ HR 3.96 [95 \% CI $1.39-11.28])$ and LVSI $(p=.014$; HR 2.87 [95 \% CI $1.25-7.10])$ remained as independent prognostic factors for LRFS.

\section{Observed acute toxicities}

Women receiving combined IVB/EBRT showed a substantially higher rate of acute gastrointestinal (GI) toxicities compared to those with sole IVB ( $37.5 \%$ vs. $8.7 \%)$. Only one patient receiving combined IVB/EBRT developed a grade 3 acute GI toxicity (enterocolitis) requiring hospitalization. Regarding acute genitourinary (GU) toxicities we observed similar rates in the IVB/EBRT and sole IVB groups (57.8 \% vs. $56.2 \%$ ). None developed acute $\mathrm{GU}$ toxicities $\geq$ grade 3 .

\section{Discussion}

The conductance of systematic LNE, especially in early stage EC, has been actively disputed over the past years [4]. Two randomized trials did not find a survival benefit from systematic pelvic LNE in EC $[5,6]$. Arguable, in 

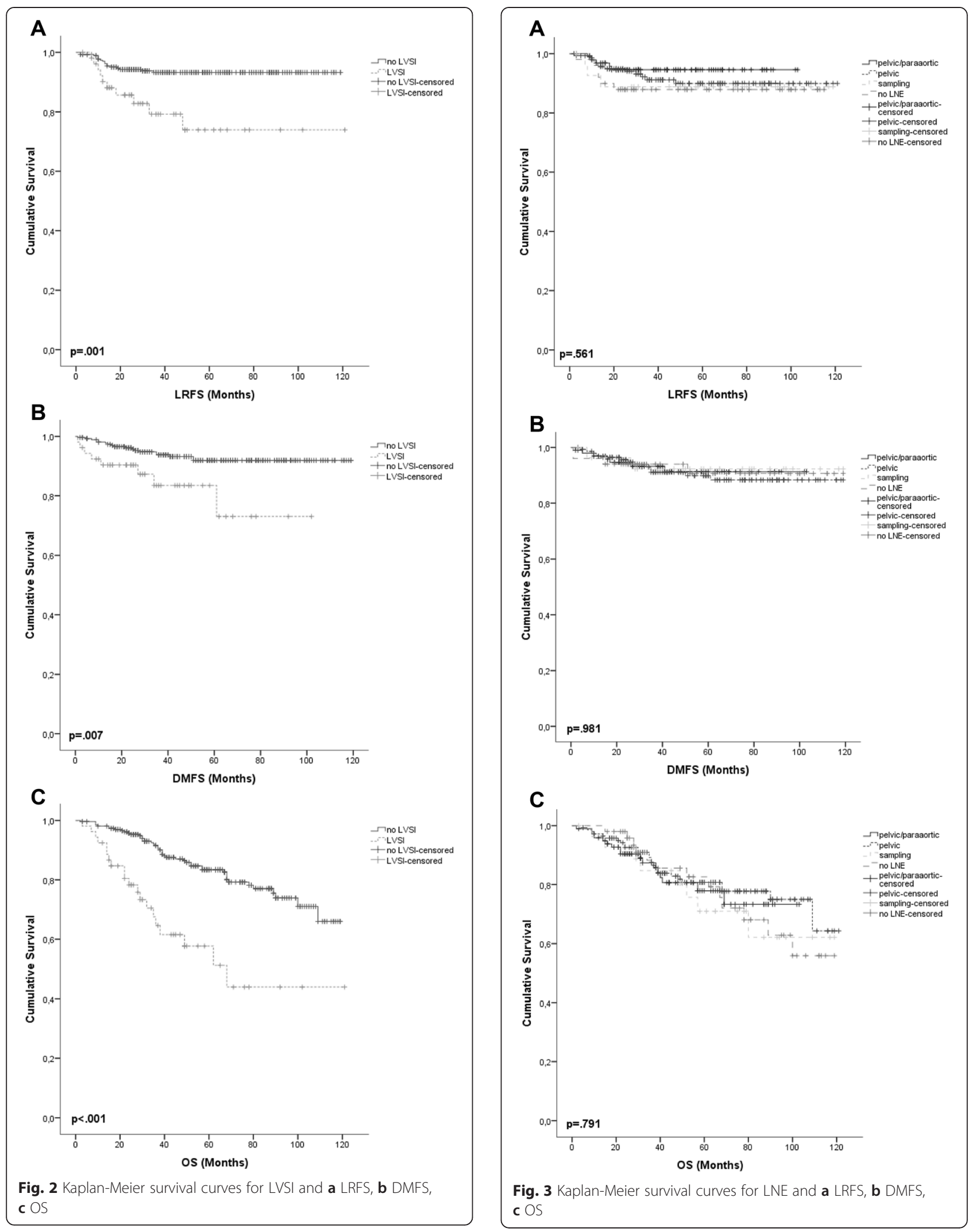
both studies paraaortic LNE was omitted, the no-LNE groups showed a high proportion of low-risk pts and adjuvant therapy was not standardized. The Japanese SEPAL trial showed a significantly prolonged OS in intermediate- and high risk patients who underwent combined pelvic and paraaortic LNE, whereas OS was not prolonged in low-risk pts [7]. The uncertainty over the therapeutic value of systematic LNE has led to a point where surgical practice differs among centers substantially and many surgeons prefer an individual risk assessment over the systematic LNE recommended in current guidelines [1, 2]. A recent report from Bogani et al. describes the clinical practice regarding LNE at the Mayo Clinic in Minnesota [8]. The colleagues from Rochester only perform systematic pelvic and paraaortic LNE in pts with $>50 \%$ myometrial invasion, nonendometrioid histology or both. Additionally paraaortic LNE is conducted when positive pelvic lymph nodes are found, while pelvic LNE is conducted based on involvement of the uterine cervix, undifferentiated grading and tumor diameter $>2 \mathrm{~cm}$. This practice seems to be supported by a recent Surveillance, Epidemiology, and End Results database analysis from Vargas et al. on risk factors for lymph node metastases in EC [9]. The cohort of women treated with adjuvant $\mathrm{RT}$ at our department was also very heterogeneous regarding surgical staging and only a minority underwent comprehensive surgical staging as demanded in current guidelines; however we were unable to detect any statistically significant benefit from surgical lymph node investigation for all 3 endpoints (LRFS, DMFS and OS) in our current study. This was surprising, since we were able to demonstrate a significantly prolonged OS after LNE in pts with type II EC previously [10]. The groups of pts with stage IIIA and IIIB as well as stage IV disease in our current study were too small for LNE- and RT-stratified subgroup analysis (Table 2), but for patients with stage I and II disease we were able to confirm these results in separate analyses. Women with intermediate risk more often received combined IVB/EBRT when LNE was omitted in our study; this, however, did not have a statistically significant influence on survival as well. Our results are generally supported by the findings of the PORTEC trials where no routine LNE was required $[11,12]$. We believe that surgical lymph node staging may be omitted in pts with type I EC in stages I-II and we further believe that women who did not receive systematic LNE and are without clinical suspicion of regional lymph node metastases may be treated as if they had undergone comprehensive surgical staging.

We found our results regarding local control, diseasefree and overall survival to generally be in line with a recent retrospective study on postoperative RT from Switzerland [13]. Several randomized trials have investigated the role of adjuvant RT in EC [11, 14-16]. All showed a significantly improved local control; even in the treatment of recurrences [17]. Since the Norwegian trial from Aalders et al. [14], published in 1980, efforts have been made to reduce RT-associated morbidity by defining subgroups of pts which do not require EBRT and may benefit from IVB alone [12, 18-22]. The PORTEC-2 trial proved that IVB alone provides similar local control rates as combined IVB/EBRT treatment in pts with higher risk profile in early stages while toxicity is substantially reduced [12, 23]. The women with FIGO stage I and stage II disease in our study cohort also had an equally good local control and OS after sole IVB and combined IVB/EBRT. Far too few patients received additional adjuvant ChT to be able to draw any conclusions on its effect on survival.

In multivariate analysis age over 66 years was associated with a shorter OS $(p=.017)$. This is in agreement with a study from Benedetti Panici et al. who reported a reduced overall and cancer specific survival in women over 65 years [24]. Other independent prognostic factors for OS in our analysis were pT stage $(p=.029)$, lymph node status $(p=.003)$ and grading $(p=.011)$. For local control we found resection status $(p=.01)$ to be an independent prognostic factor. Interestingly LVSI was the only independent prognostic factor that was found in all 3 endpoints (LRFS [ $p=.014]$, DMFS [ $p=.049]$, OS $[p=.008])$ and it was the only independent prognostic factor for DMFS. Previous studies have already reported on the prognostic relevance of LVSI in EC or considered the presence of LVSI a feature of higher risk [13-15, 25-27]. We believe that LVSI deserves more attention as a prognostic factor and that these women may be in need for a combined local and systemic treatment approach.

The observed acute GI toxicities were moderate and in line with a previous report, considering that intensity modulated radiotherapy was available only for patients treated in more recent years [28]. The relatively large proportion $(>50 \%)$ of documented acute GU toxicities in both groups is owed to the inclusion of asymptomatic grade 1 vaginal erythema.

\section{Conclusion}

Comprehensive surgical staging is rarely performed and may be omitted in women with type I EC in stages I-II. Sole IVB provides equally good local control as combined IVB/EBRT in stages I and II. Women with LVSI may be in need for a combined local and systemic therapy and LVSI should be included as a major risk factor in future randomized trials.

\section{Abbreviations}

ChT: Chemotherapy; DMFS: Distant metastases free survival; EBRT: External beam radiotherapy; EC: Endometrial cancer; HDR: High-dose rate;

IVB: Intravaginal brachytherapy; LNE: Lymphadenectomy; LRFS: Local 
recurrence free survival; LVSI: Lymphovascular space invasion; OS: Overall survival; pts: Patients; RT: Radiotherapy.

\section{Competing interests}

The authors declare that they have no competing interests.

\section{Authors' contributions}

$\mathrm{RF}$ and $\mathrm{KL}$ designed the study. RK was responsible for data collection. RF and RK performed the statistical analysis and interpretation of the results. RF drafted the manuscript. All authors critically revised and approved the final manuscript.

\section{Acknowledgement}

We acknowledge the financial support of the Deutsche Forschungsgemeinschaft and Ruprecht-Karls-Universität Heidelberg within the funding program Open Access Publishing.

\section{Received: 26 March 2015 Accepted: 9 July 2015}

Published online: 16 July 2015

\section{References}

1. Emons G, Kimmig R. Interdisciplinary S2k guidelines on the diagnosis and treatment of endometrial carcinoma. J Cancer Res Clin Oncol. 2009;135:1387-91.

2. Hernandez E. ACOG Practice Bulletin number 65: management of endometrial cancer. Obstet Gynecol. 2006;107:952-3.

3. Johnson N, Cornes P. Survival and recurrent disease after postoperative radiotherapy for early endometrial cancer: systematic review and meta-analysis. BJOG. 2007;114:1313-20.

4. Bottke D, Wiegel T, Kreienberg R, Kurzeder C, Sauer G. Stage IB endometrial cancer. Does lymphadenectomy replace adjuvant radiotherapy? Strahlenther Onkol. 2007;183:600-4.

5. Benedetti Panici P, Basile S, Maneschi F, Alberto LA, Signorelli M, Scambia G, et al. Systematic pelvic lymphadenectomy vs. no lymphadenectomy in early-stage endometrial carcinoma: randomized clinical trial. J Natl Cancer Inst. 2008;100:1707-16.

6. Kitchener H, Swart AM, Qian Q, Amos C, Parmar MK. Efficacy of systematic pelvic lymphadenectomy in endometrial cancer (MRC ASTEC trial): a randomised study. Lancet. 2009;373:125-36.

7. Todo Y, Kato H, Kaneuchi M, Watari H, Takeda M, Sakuragi N. Survival effect of para-aortic lymphadenectomy in endometrial cancer (SEPAL study): a retrospective cohort analysis. Lancet. 2010;375:1165-72.

8. Bogani G, Dowdy SC, Cliby WA, Ghezzi F, Rossetti D, Mariani A. Role of pelvic and para-aortic lymphadenectomy in endometrial cancer: Current evidence. J Obstet Gynaecol Res. 2014;40:301-11.

9. Vargas R, Rauh-Hain JA, Clemmer J, Clark RM, Goodman A, Growdon WB, et al. Tumor size, depth of invasion, and histologic grade as prognostic factors of lymph node involvement in endometrial cancer: A SEER analysis. Gynecol Oncol. 2014;133:216-20.

10. Foerster R, Kluck R, Rief H, Rieken S, Debus J, Lindel K. Survival of women with clear cell and papillary serous endometrial cancer after adjuvant radiotherapy. Radiat Oncol. 2014;9:141.

11. Creutzberg $C L$, van Putten $\mathrm{WL}$, Koper PC, Lybeert ML, Jobsen JJ, Warlam-Rodenhuis CC, et al. Surgery and postoperative radiotherapy versus surgery alone for patients with stage-1 endometrial carcinoma: multicentre randomised trial. PORTEC Study Group. Post Operative Radiation Therapy in Endometrial Carcinoma. Lancet. 2000:355:1404-11.

12. Nout RA, Smit VT, Putter H, Jurgenliemk-Schulz IM, Jobsen JJ, Lutgens LC, et al. Vaginal brachytherapy versus pelvic external beam radiotherapy for patients with endometrial cancer of high-intermediate risk (PORTEC-2): an open-label, non-inferiority, randomised trial. Lancet. 2010;375:816-23.

13. Herrera FG, Cruz OS, Achtari C, Bourhis J, Ozsahin M. Long-term outcome and late side effects in endometrial cancer patients treated with surgery and postoperative radiation therapy. Ann Surg Oncol. 2014;21:2390-7.

14. Aalders J, Abeler V, Kolstad P, Onsrud M. Postoperative external irradiation and prognostic parameters in stage I endometrial carcinoma: clinical and histopathologic study of 540 patients. Obstet Gynecol. 1980;56:419-27.

15. Keys HM, Roberts JA, Brunetto VL, Zaino RJ, Spirtos NM, Bloss JD, et al. A phase III trial of surgery with or without adjunctive external pelvic radiation therapy in intermediate risk endometrial adenocarcinoma: a Gynecologic Oncology Group study. Gynecol Oncol. 2004;92:744-51.
16. Ortoft G, Hansen ES, Bertelsen K. Omitting adjuvant radiotherapy in endometrial cancer increases the rate of locoregional recurrences but has no effect on long-term survival: the Danish Endometrial Cancer Study. Int J Gynecol Cancer. 2013;23:1429-37.

17. Sole CV, Calvo FA, Lozano MA, Gonzalez-Bayon L, Gonzalez-Sansegundo C, Alvarez $A$, et al. External-beam radiation therapy after surgical resection and intraoperative electron-beam radiation therapy for oligorecurrent gynecological cancer. Long-term outcome. Strahlenther Onkol. 2014;190:171-80.

18. Bertelsen K, Ortoft G, Hansen ES. Survival of Danish patients with endometrial cancer in the intermediate-risk group not given postoperative radiotherapy: the Danish Endometrial Cancer Study (DEMCA). Int J Gynecol Cancer. 2011;21:1191-9.

19. Chadha M, Nanavati PJ, Liu P, Fanning J, Jacobs A. Patterns of failure in endometrial carcinoma stage IB grade 3 and IC patients treated with postoperative vaginal vault brachytherapy. Gynecol Oncol. 1999;75:103-7.

20. Poulsen HK, Jacobsen M, Bertelsen K, Andersen JE, Ahrons S, Bock JE, et al. Patients with early stages of endometrial cancer should be spared adjuvant radiotherapy. Danish Endometrial Cancer Group. Ugeskr Laeger. 1997;159:3403-7.

21. Solhjem MC, Petersen IA, Haddock MG. Vaginal brachytherapy alone is sufficient adjuvant treatment of surgical stage I endometrial cancer. Int J Radiat Oncol Biol Phys. 2005;62:1379-84.

22. Micke O, Bruns F, Halek G, Schafer U, Schuck A, Willich N. Long-term results of postoperative radiotherapy for stage I endometrial carcinoma. Strahlenther Onkol. 2003;179:729-36.

23. Nout RA, Putter H, Jurgenliemk-Schulz IM, Jobsen JJ, Lutgens LC, van der Steen-Banasik EM, et al. Five-year quality of life of endometrial cancer patients treated in the randomised Post Operative Radiation Therapy in Endometrial Cancer (PORTEC-2) trial and comparison with norm data. Eur J Cancer. 2012;48:1638-48.

24. Benedetti Panici P, Basile S, Salerno MG, Di Donato V, Marchetti C, Perniola $G$, et al. Secondary analyses from a randomized clinical trial: age as the key prognostic factor in endometrial carcinoma. Am J Obstet Gynecol. 2013;210:363.e1-363.e10.

25. Bakkum-Gamez JN, Mariani A, Dowdy SC, Weaver AL, McGree ME, Martin JR, et al. Efficacy of contemporary chemotherapy in stage IIIC endometrial cancer: A histologic dichotomy. Gynecol Oncol. 2014;132:578-84.

26. Weber SK, Sauerwald A, Polcher M, Braun M, Debald M, Serce NB, et al. Detection of lymphovascular invasion by D2-40 (podoplanin) immunoexpression in endometrial cancer. Int J Gynecol Cancer. 2012;22:1442-8.

27. Weinberg LE, Kunos CA, Zanotti KM. Lymphovascular Space Invasion (LVSI) is an isolated poor prognostic factor for recurrence and survival among women with intermediate- to high-risk early-stage endometrioid endometrial cancer. Int J Gynecol Cancer. 2013;23:1438-45.

28. Barillot I, Tavernier E, Peignaux K, Williaume D, Nickers P, Leblanc-Onfroy M, et al. Impact of post operative intensity modulated radiotherapy on acute gastro-intestinal toxicity for patients with endometrial cancer: results of the phase II RTCMIENDOMETRE French multicentre trial. Radiother Oncol. 2014;111:138-43.

\section{Submit your next manuscript to BioMed Central and take full advantage of:}

- Convenient online submission

- Thorough peer review

- No space constraints or color figure charges

- Immediate publication on acceptance

- Inclusion in PubMed, CAS, Scopus and Google Scholar

- Research which is freely available for redistribution 